\title{
Antimicrobial activity and mechanism of action of Perilla essential oil against Staphylococcus aureus
}

\author{
Wenbing $\mathrm{He}^{1,4}$, Ce Shi ${ }^{2}$, Xiaoyin Long ${ }^{1}$, Xuelian $\mathrm{Liu}^{3}$ and Xingchen Zhao ${ }^{1,4^{*}}$ \\ ${ }^{1}$ Department of Food Quality and Safety, College of Food Science and Engineering, Tonghua Normal University, 134000, Tonghua, P. R. \\ China \\ ${ }^{2}$ Department of Food Quality and Safety, College of Food Science and Engineering, Jilin University, 130062, Changchun, P. R. China \\ ${ }^{3}$ College of Life Science, Tonghua Normal University, 134000, Tonghua, P. R. China \\ ${ }^{4}$ Changbai Mountain Edible Plant Resources Research and Development Engineering Center, Tonghua Normal University, 134002, \\ Tonghua, P. R. China \\ Running head: Antimicrobial activity and mechanism of PEO.
}

\begin{abstract}
Staphylococcus aureus (S. aureus) is an important cause of foodborne illness in humans and animals. In some Asian countries, Perilla (Perilla frutescens) is widely used for cooking and medicinal purposes. The current study reports its antibacterial activity against $S$. aureus. PEO exhibited significant antibacterial activity against $S$. aureus with MIC values ranged from 1 to $2 \mathrm{mg} / \mathrm{ml}$. Growth curve illustrated that PEO had time and concentration-dependent antibacterial effects against $S$. aureus. The results of this study showed that PEO exerted the inhibitory effect on $S$. aureus through cell membrane permeabilization which was associated with generalized membrane-disrupting effects, and this corresponded to a simultaneous loss of 260-nm absorbing materials. FCM assay also demonstrated that PEO treatment markedly damaged the membrane of $S$. aureus. Moreover, the SEM and TEM observations also support the above hypothesis, and strongly indicated the membrane-destructing activity of PEO. This study may contribute to the effective application of PEO as a natural antibacterial agent to control foodborne pathogens in food industries.
\end{abstract}

\section{Introduction}

Staphylococcus aureus (S. aureus) is an important and well-known foodborne pathogen due to its combination of invasiveness, antibiotic resistance, and toxin-mediated virulence [1]. Besides being the important causative agent of food poisoning by the virtue of staphylococcal enterotoxins (SEs), it is also a serious opportunistic pathogen that colonizes a third of the healthy human population and yet is the main cause of bacterial infections in the developed countries, giving rise to a variety of diseases ranging from benign skin infections to fatal infective endocarditis and necrotizing pneumonia [2]. In recent years, different multidrug-resistant strains have emerged making $S$. aureus a major concern for public health, and treatment of staphylococcal infections has traditionally been with $\beta$-lactam antibiotics. However, as time goes on, $S$. aureus developed two main mechanisms about the resistance against this kind of antibiotics. One of two main resistance mechanisms showed that the production of $\beta$-lactamases inactivating these antibiotics, which was surrounded by the development of biopharmaceutical compounds was resistant to enzymatic hydrolysis such as oxacillin and nafcillin. The other one exemplified by methicillin-resistant $S$. aureus (MRSA), resistance to methicillin is related to acquisition of SCCmec, which contains the mecA resistance gene. PBP2a which was determinant encoded by $m e c A$, is a new penicillin-binding protein with decreased affinity for methicillin and most other $\beta$-lactamases drugs [3]. The two mechanisms almost confer resistance to all $\beta$-lactamases antibiotics, with the noticeable exception of cephalosporins [4]. Therefore, it is very urgent to find a alternative and original strategy to fight against $\beta$-lactam-resistant staphylococci.

Essential oils are well-known for their beneficial biological activities including antimicrobial activities [5].

In some Asian countries, Perilla (Perilla frutescens) is widely used for cooking and medicinal purposes. Its seeds contain about $35-45 \%$ of oil, and are a good source of polyunsaturated fatty acids, especially $\alpha$-linolenic acid (ALA, C18:3)[6]. In addition, its seeds have phytosterols and tocopherols. It has been reported that increased dietary intake levels of ALA which is a precursor of eicosapentaenoic acid (EPA) and docosahexaenoic acid (DHA) can provide several beneficial effects in the health of human[7]. It has been demonstrated by conventional medicine that perilla essential oil (PEO) can be used in the treatment of depression, indigestion and certain cardiovascular diseases [8]. Except ALA, some other compounds have been identified in PEO, such as 
flavonoids, sterols, vitamin E, and phenolic compounds [9]. In addition, the antibacterial and antifungal properties of PEO were also been reported, such as antitrichophyton[10], but only a few reports illustrated its anti-S. aureus activity [11] and the inhibition of the production of staphylococcal exotoxins [12], little is known about its underlying mechanism behind the antibacterial action of PEO. This has limited the wider application of PEO as an antimicrobial agent and further research into these aspects is therefore urgent. This work investigated the chemical composition, the antibacterial activity and mechanism of PEO against $S$. aureus by several methods.

\section{Materials and methods}

\subsection{Chemical reagents, microorganisms and mediums}

PEO was extracted by water distillation and bought from the National Institute for the Control of Pharmaceutical and Biological Products (Beijing, China). The Mueller-Hinton broth (MHB) and the tryptic soy broth (TSB) were purchased from Qingdao Hope Bio-Technology Co., Ltd. (Qingdao, China). The quality control strain, ATCC 29213 was supplied by the China Medical Culture Collection Center (CMCC).

\subsection{Antimicrobial susceptibility testing of PEO}

According to the CLSI guidelines, the antimicrobial susceptibility testing of PEO on $S$. aureus was determined using the standard broth microdilution method. The minimum inhibitory concentration (MIC) was defined as the lowest concentration of drug that produced the complete inhibition of visible growth.

\subsection{Growth curves}

S. aureus strain ATCC 29213 was grown with shaking to the optical density (OD) of 0.3 at $600 \mathrm{~nm}$ in MHB, and then was distributed as 100-ml volumes into four 250-ml Erlenmeyer flasks. Bacteria were grown in the presence of increasing concentrations of PEO $(1 / 2 \times \mathrm{MIC}, 1 \times$ MIC and $2 \times$ MIC) at $37{ }^{\circ} \mathrm{C}$ with shaking at $200 \mathrm{rpm}$ under aerobic conditions, and the growth of cell was measured by reading the OD values at $600 \mathrm{~nm}$ at different time points (Xing et al., 2012).

\subsection{Integrity of cell membrane}

The cell membrane integrity of $S$. aureus ATCC 29213 was assessed by measuring the release of cell constituents including proteins and nucleic acids into the suspension according to the method described by Du et al. [13], with some modifications. Bacterial cells from the $100 \mathrm{ml}$ working culture were centrifuged at $5000 \mathrm{rpm}$ for $15 \mathrm{~min}$, washed three times using $0.1 \mathrm{M}$ phosphate buffer solution (PBS, pH 7.4), and then resuspended in PBS. The final cell suspension of tested microorganisms was adjusted to the OD values at $420 \mathrm{~nm}$ of 0.7 . Then, $100 \mathrm{ml}$ of cell suspension treated with different concentrations $(1 / 2 \times$ MIC, $1 \times$ MIC and $2 \times$ MIC) PEO were incubated at $37^{\circ} \mathrm{C}$ with shaking for $1 \mathrm{~h}, 2 \mathrm{~h}, 4 \mathrm{~h}, 6 \mathrm{~h}$ and $8 \mathrm{~h}$. Untreated cell suspension was used as the control group, control flask without PEO was tested similarly. Next, the suspensions were collected by centrifuging at $6000 \mathrm{~g}$ for $5 \mathrm{~min}$, diluted with PBS, and the absorption at $260 \mathrm{~nm}$ was measured using an UV-vis spectrophotometer (Shimadzu, Tokyo, Japan).

\subsection{Membrane permeability assay}

Mmbrane permeability of bacterial cells (S. aureus ATCC 29213) was investigated by propidium iodide (PI) staining using flow cytometry (FCM). PI shows substantially increased fluorescences on binding to intracellular nucleic acids, and the dye normally bear two positive charges and are excluded from cells with intact membranes, while they stain nucleic acids in cells with damaged membranes (Haugland, 1996). In brief, bacteria were frstly incubated with PEO at different concentrations $(1 / 2 \times \mathrm{MIC}, 1 \times \mathrm{MIC}$ and $2 \times \mathrm{MIC})$ for 3 h. Control group without PEO treatment was tested similarly. After photodynamic treatment, bacteria were harvested (at $4000 \mathrm{rpm}, 5 \mathrm{~min})$ and PI $(10 \mu \mathrm{g} / \mathrm{ml})$ was added. After the incubation in the dark for $20 \mathrm{~min}$, membrane permeability of the stained cells was analyzed using a FCM with the excitation of the light at the wavelength of $488 \mathrm{~nm}$.

\subsection{Scanning electron microscopy (SEM) of S. aureus strains exposed to PEO}

Logarithmic phase bacteria were collected, then adhere to polylysine-coated coverslips for approximately $12 \mathrm{~h}$ and treated with PEO at $1 / 2 \times$ MIC, $1 \times$ MIC and $2 \times$ MIC for $3 \mathrm{~h}$. Then cells were washed with PBS buffer after incubation and fixed in $2.5 \%$ glutaraldehyde at $4{ }^{\circ} \mathrm{C}$ for about $2 \mathrm{~h}$. Bacterial cells were washed in PBS and were post-fixed with osmium tetroxide in $0.1 \mathrm{M}$ of cacodylate buffer ( $\mathrm{pH}$ 7.2) for $30 \mathrm{~min}$. After this, the cells were dehydrated using sequential exposure per ethanol concentrations $(30 \%-100 \%)$ and the ethanol was replaced by tertiary butyl alcohol at last. The bacterial cells were freezedried with the vacuum freeze drier (Hitachi ES-2030), coated with the ion sputtering apparatus (Hitachi E-1010), and observed by SEM (Hitachi S-3400 N). Untreated cells were used as the control group and were similarly processed.

\subsection{Transmission electron microscopy (TEM) of S. aureus strains exposed to PEO}

The preparation of samples treated with PEO in TEM was the same as that in SEM analysis, and TEM analysis was performed according to method in the literature with some modifications. In brief, after incubation, the pellets were collected by centrifugating at $11,000 \times \mathrm{g}$ for $10 \mathrm{~min}$, and fixed with $4 \%$ glutaraldehyde overnight at $4{ }^{\circ} \mathrm{C}$. To perform TEM analysis, the pellets were subjected to a 
series of treatments according to the guidelines.

\subsection{Statistical analysis}

All samples were analyzed in triplicate, and the data are presented as the mean values \pm standard deviations (SD) $(\mathrm{n}=3)$. Independent Student $\mathrm{t}$ tests were used to determine statistical differences, and $\mathrm{p} \leq 0.05$ was considered significant.

\section{Results and discussion}

\subsection{Effect of PEO on S. aureus growth}

In our study, PEO exhibited a significant antibacterial activity against ten food-borne isolates of $S$. aureus and one quality control strains, ATCC 29213, with MIC values of $1-2 \mathrm{mg} / \mathrm{ml}$, which have been reported by our previous study [17]. The MIC value of PEO against $S$. aureus strain ATCC 29213, which was selected for further experiments, was $2 \mathrm{mg} / \mathrm{ml}$. It has been reported that the MIC value of PEO against $S$. aureus ATCC 6538 was $5 \mu \mathrm{l} / \mathrm{ml}(\sim 5 \mathrm{mg} / \mathrm{ml})$ [11] which was a little higher than that calculated in the present study, and Qiu et al. [12] also indicated that PEO exhibited a significant antibacterial effect against the test $S$. aureus strains, with MIC values ranging 0.2 to $0.8 \mu 1 / \mathrm{ml}(\sim 0.2-0.8 \mathrm{mg} / \mathrm{ml})$.

The growth curve of S. aureus ATCC 29213 in Fig.1 shows that the OD values of the culture increase steadily with $1 \mathrm{mg} / \mathrm{ml}(1 / 2 \times \mathrm{MIC})$ and $2 \mathrm{mg} / \mathrm{ml}(1 \times \mathrm{MIC})$ PEO treatment, though at $4 \mathrm{mg} / \mathrm{ml}(2 \times \mathrm{MIC})$ of PEO bacteria showed almost no growth. Within $30 \mathrm{~min}$ of PEO treatment, no obvious differences in the OD values could be observed for any of the cultures. With 1 and $2 \mathrm{mg} / \mathrm{ml}$ of PEO treatment, the OD values increased at a significantly slower rate than those observed for the control group. At $8 \mathrm{~h}$, the OD values of the cultures treated with 1,2 and $4 \mathrm{mg} / \mathrm{ml}$ of PEO were around $50.4 \%$, $60.0 \%$, and $83.7 \%$ of the OD of the control group, respectively. The results illustrate that subinhibitory concentrations of PEO can efficiently inhibit the growth of S. aureus ATCC 29213.

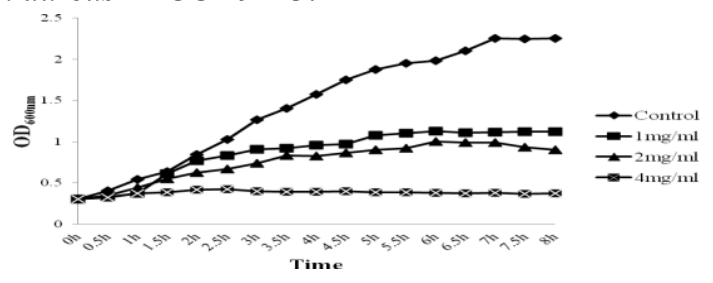

Fig. 1. Growth curve of S. aureus ATCC 29213 after exposure to various concentrations of PEO.

\subsection{Integrity of cell membrane}

Intracellular constituents such as DNA and RNA have strong UV absorption at $260 \mathrm{~nm}$, and can be released into the extracellular when cell membrane is destructed during the challenge of antimicrobial drugs [18]. Thus, the UV absorption value can show the degree of cell membrane damage. Fig.2 demonstrated the results when
S. aureus were treated with different concentrations of PEO from $1 \mathrm{~h}$ to $8 \mathrm{~h}$. The results indicated that after treating with PEO, the release of cell constituents increased significantly with the increased concentrations of PEO, while no obvious changes in the OD values of control group cells of $S$. aureus ATCC 29213 was observed. When comparing with the control group, the value $\mathrm{OD}_{260 \mathrm{~nm}}$ of suspensions treated with $1 / 2 \times \mathrm{MIC}$ PEO increased from 0.124 at $0 \mathrm{~h}$ to 2.204 at $8 \mathrm{~h}$. While they increased from 0.136 at $0 \mathrm{~h}$ to 2.712 when treatment at $1 \times$ MIC. Furthermore, after treatment with $2 \times$ MIC PEO, the absorbance value at $\mathrm{OD}_{260 \mathrm{~nm}}$ increased significantly from 0.142 at $0 \mathrm{~h}$ to 3.194 at $8 \mathrm{~h}$. After $1 \mathrm{~h}$ of treatment at $2 \times$ MIC, approximately 2 -fold increase was observed in the OD value of the cell culture filtrates compared to the $1 / 2 \times$ MIC sample. The result directly indicates the leakage of 260 -nm absorbing constituents from the bacterial cells treated with PEO, and the damage of cell membranes was concentration-dependent.

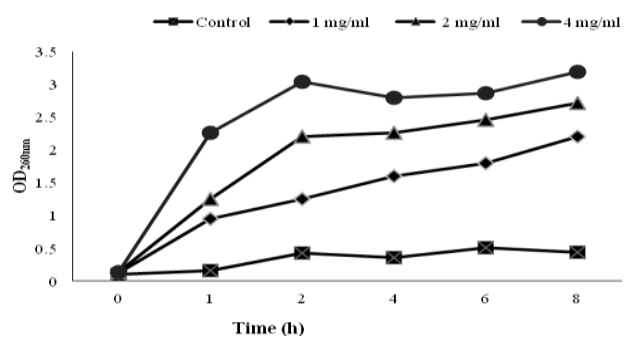

Fig.2. Effect of PEO on the release rate of 260-nm absorbing material from S. aureus ATCC 29213 at different time points.

The bacterial cell's macromolecules including nucleic acids such as DNA and RNA, residing throughout the interior of the cell (in the cytoplasm), are the key structural components. The transfer of intracellular information by the processes of translation, transcription and DNA replication occur in the same compartment and can interact with other cytoplasmic structures. The measurement of some cell leakage markers including $260-\mathrm{nm}$ absorbing materials is indicative of bacterial cell membrane sensitivity to some antibacterial agent related to unexposed cells [19]. As one of the results of antibacterial action, the DNA replication machinery becomes arrested at blocked replication forks, resulting in inhibition of DNA synthesis, which immediately results in bacteriostasis and eventually cell death[20]. In addition, the peptidoglycan layer of the cell wall of $S$. aureus consists of networks with lots of pores, so foreign molecules could easily enter the cell. Since that $S$. aureus does not have the outer membrane composed of lipopolysaccharide, lipoprotein and phospholipids to prevent the influx of foreign molecules or the release of intracellular constituents, it will cause $S$. aureus more sensitive than Gram-negative bacteria to exogenous agents [18].

\subsection{Membrane permeability assay}

To determine whether PEO is causing membrane instability, we added PI to the treated cells, because PI is a positively charged nucleic acid binding dye and gives 
red fluorescence at $488 \mathrm{~nm}$ excitation after getting associated with DNA. Hence, after PEO treatment, the cells of $S$. aureus were observed under FCM for permeabilization. As shown in Fig.3, the results revealed an increase in the PI fluorescence after PEO treatment as the concentration increasing in comparison to untreated cells (in the area of R2), and the presence of PI fluorescence confirmed that permeabilization is occurring in $S$. aureus cells. In the control group, only a small proportion was stained with PI, the positive rate of dead bacterial cells was $0.23 \%$ (in the area of R2). However, after treatment with $1 / 2 \times$ MIC PEO and $1 \times$ MIC PEO, FCM showed that the positive rate of dead bacterial cells stained by PI increased to $80.05 \%$ and $86.83 \%$ respectively (in the area of $\mathrm{R} 2$ ). In addition, most of the population was stained with PI after exposure to PEO at $2 \times \mathrm{MIC}$, the proportion of membrane damaged cells increased to $95.76 \%$ (in the area of R2).

It is considered that FCM technique can provide a good evaluate of the membrane integrity of various bacteria. Membrane integrity is a key factor to the barrier function of the cell membrane, which plays an important role in keeping optimal internal conditions for energy transduction and metabolism [21]. Therefore, even there is only relatively slight damage to the structural integrity of the cell membrane, but it could detrimentally affect cell metabolism and lead to cell growth inhibition and eventually cell death[22]. These results illustrated that PEO caused a significant destruction on the membrane integrity of $S$. aureus, which was in accordance with the results of the release of 260-nm absorbing materials assay, demonstrating that the primary target of PEO in its antibacterial action might be the cell membrane. (a)

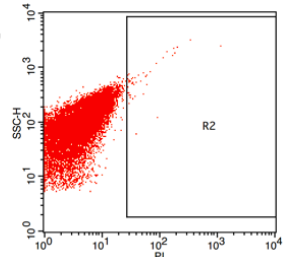

(c)

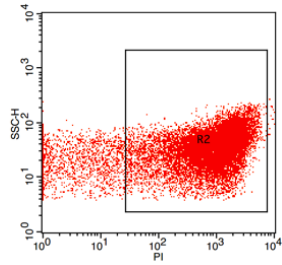

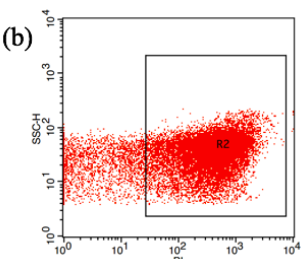

(d)

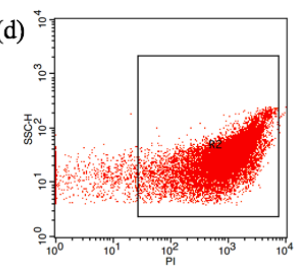

Fig. 3. Membrane permeability of S. aureus ATCC 29213 was measured using flow cytometry with PI staining after treatment with PEO at different concentrations for $3 \mathrm{~h}$. (a) Control (b) Treatment with PEO at $1 / 2 \times$ MIC (c) Treatment with PEO at 1 $\times$ MIC (d) Treatment with PEO at $2 \times$ MIC.

\subsection{Electron microscopy observation}

In order to search for some related clues to possible antimicrobial mechanisms of PEO on $S$. aureus, TEM and SEM were performed on $S$. aureus, treated with PEO for $3 \mathrm{~h}$. The results of electron micrographs illustrated a remarkable modification of bacterial cell shape after treated with $\mathrm{PEO}$ in different concentrations for $3 \mathrm{~h}$. Although numerous bacterial cells were observed, only some representative cells have been showed in Fig. 5 and Fig 6.

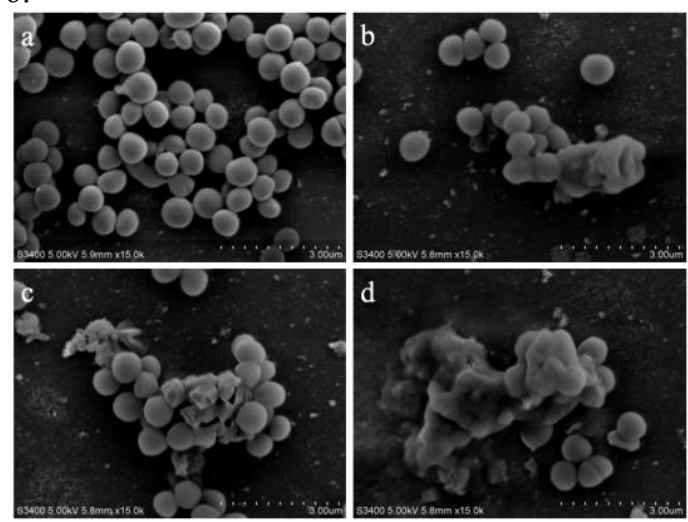

Fig. 4. SEM observation of S. aureus ATCC 29213 exposed $\mathrm{PEO}$ at different concentrations for $3 \mathrm{~h}$. (a) Control (b) Treatment with PEO at $1 / 2 \times$ MIC (c) Treatment with PEO at 1 $\times$ MIC (d) Treatment with PEO at $2 \times$ MIC.

The surface morphology in the PEO treated $S$. aureus cells was visualized by SEM (Fig. 4). The surfaces of $S$. aureus cells treated with PEO went through evident morphological changes compared with the untreated $S$. aureus cells (the control group). The untreated $S$. aureus cells were typically rod shaped, and remained intact with no visible change in the integrity of cell surface (Fig. 4a), whereas some cells exposed to PEO became pitted, deformed, shriveled, adhesive together and even broken (Fig. 4, b, c and d), which may lead to the leaking of genetic and nutrient materials. In addition, the changes were more obvious, frequent with the increase of treatment time and concentration of PEO. The electron micrographs supported the results of the integrity and permeability of bacterial cell membrane assays, and demonstrated that PEO may have strong effects on the cell wall and cytoplasmic membrane. It has been reported that essential oils and their chemical compositions (such as terpenes and their related alcohols) show their antimicrobial activity against various cell parts, especially the cytoplasm and membrane, and sometimes completely change the morphology of cells[23]. Therefore, the results of altered morphology of $S$. aureus exposed to PEO revealed that the cytoplasmic membrane was compromised. TEM of untreated S. aureus (Fig. 5a) illustrated a typical structure of Gram-positive bacteria: uniform cytoplasm surrounded by cytoplasm membrane which tightly adhered to cell wall. After treated for $3 \mathrm{~h}$ with $1 / 2 \times$ MIC PEO (Fig. 5b), it still remained regular outline and cytoplasm membrane tightly adhered to cell wall, but the density of cytoplasm became rare. After incubation with the MIC and $2 \times$ MIC PEO, a wide range of cellular pathologies and extensive ultrastructural damages were observed. After treated with MIC, cytoplasm became more rarefied and even appeared blank (Fig. 5c). When treated with $2 \times \mathrm{MIC}$, obvious pores were formed for $S$. aureus, it was also noticed that some lysed cells with severely damaged or depleted cell content and with a lamellar form of the cytoplasm were appeared (Fig. 5d). A large number of previous TEM analyses illustrated that the antimicrobial effects of natural products on the bacterial cell morphology were 
diverse, including the formation of pores on the cell membrane [24], leakage of cytoplasmic materials [25], appearance of ghost-like bacteria, and complete cell lysis [26]. When treated with PEO, lysis of the cell envelope and leakage of cytoplasmic contents were monitored in the treated $S$. aureus cells. In addition, the dense granules or tightly condensed substances accumulated around the cell surface were assumed to be the deposition of the cytoplasmic proteins of inactivated bacteria [27]. Based on this, PEO might target different parts of the bacterial cell, and the antimicrobial activity of PEO could not be attributed to one specific mechanism. Therefore, further research need to be done to investigate the effect of PEO on intracellular macromolecules or bacterial pathways and provide more information to fully understand the mode of antibacterial action of PEO.
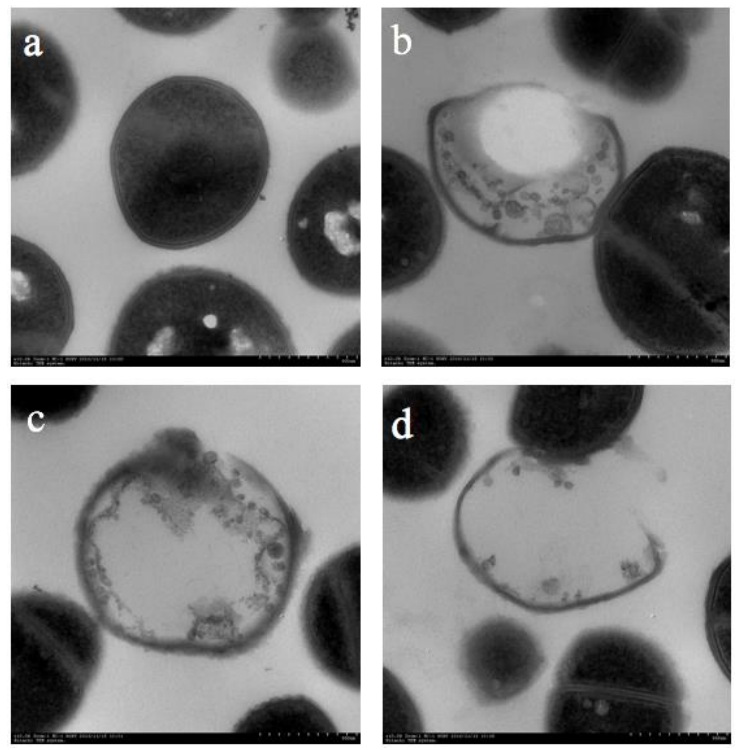

Fig. 5. TEM observation of $S$. aureus ATCC 29213 exposed t PEO at different concentrations for $3 \mathrm{~h}$. (a) Control (b) Treatment with PEO at $1 / 2 \times$ MIC (c) Treatment with PEO at 1 $\times$ MIC (d) Treatment with PEO at $2 \times$ MIC.

Besides, most compounds identified by GC-MS in our study are monoterpene hydrocarbons, oxygenated monoterpenes, sesquiterpene hydrocarbons and oxygenated sesquiterpenes, it has been reported that monoterpene or sesquiterpene hydrocarbons and their oxygenated derivatives exhibit potential antimicrobial activities [28]. Moreover, Gill, Delaquis, Russo, \& Holley[29] have revealed that whole essential oils have a greater antibacterial activity than the mixed major components and they also indicated that minor compounds may have synergistic effects and are crucial for the antibacterial activity. Thus, PEO is more promising as a natural food preservative for the control of bacteria in commercial applications than single compounds. Moreover, whole essential oils may be able to fight resistant strains of microorganisms due to their different modes of action.

\section{Conclusion}

In brief, the current study reports the chemical composition of PEO along with its antibacterial activity against $S$. aureus. The PEO exhibited significant antibacterial activity against $S$. aureus with MIC values ranged from 1 to $2 \mathrm{mg} / \mathrm{ml}$. Growth curve illustrated that PEO had time- and concentration-dependent antibacterial effects against $S$. aureus. The results of this study indicated that the PEO exerted its inhibitory effect through cell membrane permeabilization which was associated with generalized membrane-disrupting effects, and this corresponded to a simultaneous loss of 260-nm absorbing materials. FCM assay also demonstrated that PEO treatment markedly damaged the membrane of $S$. aureus. Moreover, the SEM and TEM observations also support the above hypothesis, and strongly indicated the membrane activity of PEO. This should lead to effective application of PEO as a kind of natural antimicrobial agent to control foodborne pathogens in food industries.

\section{Acknowledgments}

Financial support for this work came from the following sources: the Foundation of the Education Department of Jilin Province in 2018 (JJKH20180863KJ).

\section{References}

1. M. Á. Argudín, M. C. Mendoza and M. R. Rodicio, Food poisoning and Staphylococcus aureus enterotoxins. Toxins, 2, 1751-1773, (2010).

2. J. Haaber, J. R. Penadés and H. Ingmer, Transfer of antibiotic resistance in Staphylococcus aureus. Trends in Microbiology, 25, 893-905, (2017).

3. F. C. Tenover, Mechanisms of antimicrobial resistance in bacteria. The American journal of medicine, 34, S3-S10, (2006).

4. E. Bouza, New therapeutic choices for infections caused by methicillin-resistant Staphylococcus aureus. Clinical Microbiology and Infection, 15, 44-52, (2009).

5. F. Bakkali, S. Averbeck, D. Averbeck and M. Idaomar, Biological effects of essential oils -A review. Food and Chemical Toxicology, 46, 446-475, (2008).

6. H. K. Kim, S. Choi and H. Choi, (Suppression of hepatic fatty acid synthase by feeding $\alpha$-linolenic acid rich perilla oil lowers plasma triacylglycerol level in rats. The Journal of nutritional biochemistry, 15, 485-492, 2004).

7. J. Lee and Y. O. Song, Perilla oil rich in $\alpha$-linolenic acid suppresses hepatic SREBPs and NF- $\kappa$ B expression in hypercholesterolemia-induced apolipoprotein E knockout mice. Food Science and Biotechnology, 21, 807-813, (2012).

8. H. Jo, M. O. Kim, J. Lee, H. Kim and Y. O. Song, Alti-atherogenic properties of emulsified perila oil (EPO) in apoE KO mice and plasma lipid lowering effects of rice porridge containing EPO in healthy young adults. Food Science and Biotechnology, 22, 79-85, (2013).

9. J. H. Lee, H. P. Ki, M. H. Lee, H. T. Kim, W. D. Seo, 
J. Y. Kim, et al. Identification, characterisation, and quantification of phenolic compounds in the antioxidant activity-containing fraction from the seeds of Korean perilla (Perilla frutescens) cultivars. Food Chemistry, 136, 843-852, (2013).

10. S. Inouye, Y. Nishiyama, K. Uchida, Y. Hasumi, H. Yamaguchi and S. Abe, The vapor activity of oregano, perilla, tea tree, lavender, clove, and geranium oils against a Trichophyton mentagrophytes in a closed box. Journal of Infection and Chemotherapy, 12, 349-354, (2006).

11. F. Lv, H. Liang, Q. Yuan and C. Li, In vitro antimicrobial effects and mechanism of action of selected plant essential oil combinations against four food-related microorganisms. Food Research International, 4, 3057-3064, (2011).

12. J. Qiu, X. Zhang, M. Luo, H. Li, J. Dong, J. Wang, et al. Subinhibitory concentrations of Perilla oil affect the expression of secreted virulence factor genes in Staphylococcus aureus. PLoS One, 6, e16160, (2011).

13. W. Du, C. Sun, Z. Liang, Y. Han and J. Yu, Antibacterial activity of hypocrellin A against Staphylococcus aureus. World Journal of Microbiology and Biotechnology, 28, 3151-3157, (2012).

14. J. LiuY. K., Wan, Z. Z. Zhao and H. B. Chen, Determination of the content of rosmarinic acid by HPLC and analytical comparison of volatile constituents by GC-MS in different parts of Perilla frutescens (L.) Britt. Chemistry Central Journal, 7, 1-11, (2013).

15. C. You, Y. Wang, W. Zhang, K. Yang, Y. Wu, Z. Geng, et al. Chemical constituents and biological activities of the Purple Perilla essential oil against Lasioderma serricorne. Industrial Crops and Products, 61, 331-337, (2014).

16. B. K. Huang, Y. L. Lei, Y. H. Tang, J. C. Zhang, L. P. Qin and J. Liu, Comparison of HS-SPME with hydrodistillation and SFE for the analysis of the volatile compounds of Zisu and Baisu, two varietal species of Perilla frutescens of Chinese origin. Food Chemistry, 125, 268-275, (2011).

17. X. Zhao, C. Shi, R. Meng, Z. Liu, Y. Huang, Z. Zhao, et al. Effect of nisin and perilla oil combination against Listeria monocytogenes and Staphylococcus aureus in milk. Journal of Food Science and Technology, 53, 2644-2653, (2016).

18. C. Z. Chen and S. L. Cooper, Interactions between dendrimer biocides and bacterial membranes. Biomaterials, 23, 3359-3368, (2002).

19. V. K. Bajpai, A. Sharma and K. H. Baek, Antibacterial mode of action of Cudrania tricuspidata fruit essential oil, affecting membrane permeability and surface characteristics of food-borne pathogens. Food Control, 32, 582-590, (2013).

20. M. A. Kohanski, D. J. Dwyer and J. J. Collins, How antibiotics kill bacteria: from target to networks.
Nature Reviews Microbiology, 8, 423-435, (2010).

21. E. Sánchez, S. García and N. Heredia, Extracts of edible and medicinal plants damage membranes of Vibrio cholerae. Applied Environmental Microbiology, 76, 6888-6894, (2010).

22. S. D. Cox, C. M. Mann, J. L. Markham, J. E. Gustafson, J. R. Warmington and S. G. Wyllie, Determining the antimicrobial actions of tea tree oil. Molecules, 6, 87-91, (2001).

23. C. F. Carson, B. J. Mee and T. V. Riley, Mechanism of action of Melaleuca alternifolia (tea tree) oil on Staphylococcus aureus determined by time-kill, lysis, leakage, and salt tolerance assays and electron microscopy. Antimicrobial Agents Chemotherapy, 46, 1914-1920, (2002).

24. S. Y. Lee, S. I. Lim and D. H. Ahn, Antibacterial mechanism of Myagropsis myagroides extract on Listeria monocytogenes. Food Control, 42, 23-28, (2014).

25. J. R. Bai, Y. P. Wu, X. Y. Liu, K. Zhong, Y. N. Huang and $\mathrm{H}$. Gao, Antibacterial activity of shikimic acid from pine needles of Cedrus deodara against Staphylococcus aureus through damage to cell membrane. International Journal of Molecular Sciences, 16, 27145-27155, (2015).

26. D. Teng, X. Wang, D. Xi, R. Mao, Y. Zhang, Q. Guan, et al. A dual mechanism involved in membrane and nucleic acid disruption of avbd103b, a new avian defensin from the king penguin, against Salmonella enteritidis cvec3377. Applied Environmental Microbiol. 98, 8313-8325, (2014).

27. Q. L. Feng, J. Wu, G. Q. Chen, F. Z. Cui, T. N. Kim and Kim, J. O. A mechanistic study of the antibacterial effect of silver ions on Escherichia coli and Staphylococcus aureus. Journal of Biomeddical Materials Research, 52, 662-668, (2000).

28. I. M. Helander, A. Hanna-Leena, K. Latva-Kala, T. Mattila-Sandholm, I. Pol, E. J. Smid, et al. Characterization of the action of selected essential oil components on gram-negative bacteria. Journal of Agricultural and Food Chemistry, 46, 3590-3595, (1998).

29. A. O. Gill, P. Delaquis, P. Russo and R. A. Holley, Evaluation of antilisterial action of cilantro oil on vacuum packed ham. International Journal of Food Microbiology. 73, 83-92, (2002). 